\title{
Parametric effects of blocking and winning in a competition paradigm of human aggression
}

\author{
MICHAEL HYNAN, SUZANNE HARPER, CYNTHIA WOOD, and CAROL KALLAS \\ University of Wisconsin, Milwaukee, Wisconsin 53201
}

\begin{abstract}
Two experiments were conducted to determine the effects of parametric manipulations of blocking and winning upon human aggression in a competitive game. Both manipulations affected the number of blocks delivered by subjects, but neither manipulation influenced shocking. The failure to find aggression effects was attributed to the low levels of nonarbitrary frustration produced by the manipulations. Mood data from Experiment 1 confirmed the validity of the shock response in the competition paradigm.
\end{abstract}

This paper describes two experiments designed to investigate the effects of two parametric manipulations upon performance in a new paradigm of human aggression. The new procedure (Hynan \& Esselman, Note 1) was designed to investigate the influence of the target upon the aggressor in a face-to-face interaction. In the procedure, the subject engages in a manual game with a research assistant (target). The goal is the accumulation of points scored by pressing a telegraph key. In addition to scoring points, each competitor can temporarily block the point accumulation of the opponent, and the subject can also shock the assistant (the aggressive response).

\section{EXPERIMENT 1}

In order to obtain a better understanding of the variables controlling shocking and blocking by subjects, six schedules of blocking were administered to groups of subjects. Four of the groups received $0,12,24$, or 48 noncontingent blocks, delivered on a randomly derived schedule. A fifth group (R) received only retaliatory blocks (i.e., these subjects were blocked only if they delivered a block). The sixth group $(12+R)$ received the schedule used by Hynan and Esselman (Note 1): 12 noncontingent blocks plus retaliatory blocks. Since blocking can be considered a frustrating event (Buss, 1963), it was expected that levels of frustration and aggression would be positively related to the number of blocks received.

\section{Method}

Subjects. Data were obtained from 30 male and 30 female undergraduates from the University of Wisconsin-Milwaukee.

Apparatus. The subject and opponent were seated at opposite sides of a table. The game was housed in a $60 \times 31 \times 30 \mathrm{~cm}$ box on

All authors participated in the design of Experiment 1. Experiment 2 was part of the masters' thesis of the second author. Reprint requests should be sent to Michael T. Hynan, Department of Psychology, University of Wisconsin-Milwaukee, P.O. Box 413, Milwaukee, Wisconsin 53201. We would like to acknowledge the help of those who served as research assistants: Trilby Arnold, Becky Boone, Eric Constantz, and Judy Esselman. top of the table. The subject's panel contained a block lever on the right, a telegraph key at the lower center, and a shock button on the left. Two counters (the scoreboard) with adjacent lights were positioned above the telegraph key. The research assistant's panel was almost identical; it lacked only the shock button. A start button and green lamp were on top of the box. A Lafayette ac shock source with current meter was on the right of the game box, facing the subject. Shock was delivered through finger electrodes attached to the assistant's left hand.

Subjects filled out a modified version of the Profile of Moods State (POMS) constructed by McNair, Lorr, and Droppleman (1971). The POMS contains a number of adjectives answered on a 5-point scale. The POMS was scored on the anger and tension scales. Subjects also responded to three additional adjectives (frustrated, thwarted, and disappointed), which composed a frustration scale. Three female college students served as assistants. (Past research in our laboratory has shown that male and female targets are shocked equivalently.)

Procedure. Subjects were informed that the experiment studied the emotional effects of competition. Prior to the game, the target was introduced as a research assistant experienced at the game. Assistants behaved in a neutral fashion. Each game was a race to score 900 points. Each competitor scored points by repeatedly pressing his respective telegraph key. Both competitors could block, a response that prevented the accumulation of points by the opponent for $5 \mathrm{sec}$. During a block, the light next to the blocked competitor's counter was lit. Only subjects could shock, and subjects were told that shocking might compensate for the assistant's experience. Shocks delivered during the game were received by the targets. The experimenter self-administered a . .5 -sec . 4-mA shock (this duration and intensity were constant throughout) and invited subjects to do so. The instructions stressed that while blocking and shocking were optional, subjects should engage in these responses if they felt like it.

The green lamp was on while taped instructions were played and was extinguished at the start of the game. Then the assistant placed her left hand on the start button and said, "One, two, three." At "three," the assistant pressed the start button, and both competitors began to respond. Subjects filled out the POMS and the frustration scale during the second of two rest periods during the game.

When the assistant pressed the start button, electromechanical equipment pulsated the assistant's counter on a variable-time (VT) 8.8-point/sec schedule with a range of 6.1-11.5 points/sec. During this time, the assistant rapidly pressed the telegraph key. Just before a block was to be delivered to a subject, the light next to the upper counter on the assistant's panel was illuminated for $.35 \mathrm{sec}$. In response, the assistant stopped tapping on the telegraph key and made a block response. Thus the assistant was only mimicking what was electronically programmed. Both subject and assistant were limited to the use of their right hands. 


\section{Results}

The probability of winning was related to the block manipulation $\left[\chi^{2}(5)=16.8, p<.01\right]$. This is illustrated in the right-hand panel of Figure 1. Subjects in the four noncontingent conditions won more often than retaliatory subjects did, and in direct proportion to the number of blocks received. The nominal criterion of shocking was not related to the block manipulation, and shock was used by 11 males and 6 females (nonsignificant).

Shock frequency and block frequency scores were analyzed in a 2 (sex) by 6 (block condition) by 3 (game period) multivariate analysis of variance. There was only one significant multivariate effect, the block manipulation $[\mathrm{F}(10,94)=2.48, \mathrm{p}<.05]$. Univariate follow-ups showed that the frequency of blocking by subjects was related to the block manipulation $[F(5,48)=4.48, p<.01]$. Tukey h.s.d. multiple comparisons (Kirk, 1968) showed that there were significant differences in block frequency between all pairs of groups (with the exception of the comparison between the 0-block group and R) (ps $<.05)$. The block frequency data in the left panel of Figure 1 illustrates the positive correlation between the number of blocks received and those delivered.

A separate 2 (sex) by 6 (block condition) multivariate analysis of variance was applied to the three mood-state measures (anger, tension, and frustration). None of these tests was significant, and, overall, subjects rated themselves as being "a little frustrated" (mean $=.84$ ). Subjects who shocked described themselves as being both more angry $[\mathrm{r}(58)=.36$, $\mathrm{p}<.01]$ and more tense $[\mathrm{r}(58)=.40, \mathrm{p}<.01]$ than those who did not shock. Also, the score difference after the second game period (subject's points minus assistant's points) was related to self-reported frustration $[\mathrm{r}(58)=-.29, \mathrm{p}<.05]$ and tension $[\mathrm{r}(58)=$ $.30, \mathrm{p}<.05]$.

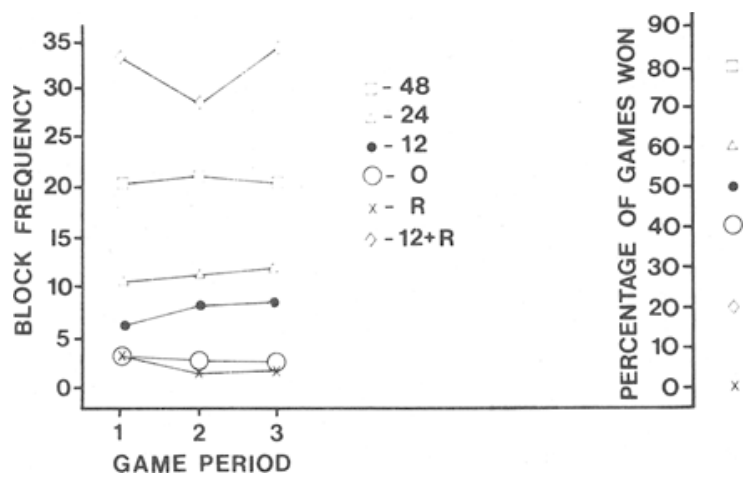

Figure 1. The left panel shows mean block responses for the six block conditions across the three game periods. The right panel shows the percentage of games won by subjects in the six block conditions.

\section{Discussion}

The frequency of blocking by subjects was dependent upon and directly related to the number of blocks received. This modeling was potent and exerted more control over blocking than another possible determinant, winning. Blocking by subjects in the nonretaliatory conditions was instrumental in winning, yet the only subjects who won consistently were those exposed to an assistant who blocked often. Subjects who received few blocks could have won easily if they had blocked often. However, matching the low rate of blocking by their opponents precluded this. Data from the two retaliatory conditions also support the role of modeling. Subjects in the $\mathbf{R}$ group blocked at a very low rate because they did not receive noncontingent blocks. Subjects in the $12+R$ group displayed the highest rate of blocking. These subjects often matched the assistant after one or more noncontingent blocks were delivered, and then they received retaliatory blocks. This further escalated the frequency of blocking by assistants, which was subsequently modeled.

The block manipulation failed to influence both the frustration of the subjects and their aggressiveness. There are two possible reasons for this. First, receiving blocks was not the only theoretical source of frustration. By looking at the scoreboard, subjects were continuously informed of the chances of winning. Because two potential sources of frustration (the number of blocks received and the chances of winning) were inversely related, frustration level may have been equivalent in the four noncontingent groups. However, in the two retaliatory groups, there should have been differences in the level of frustration. Subjects in both groups seldom won, but they experienced differences in the number of blocks received. Subjects in $12+R$ should have experienced the most frustration in the experiment, and more subjects $(50 \%)$ in $12+\mathrm{R}$ did aggress, compared with other groups, but this difference was not significant. A second account of the failure of the block manipulation was that only low levels of frustration were produced in all groups. One source of the low level of frustration lies in the nature of the block response. Because the assistant had some justification in blocking, a block can be considered a nonarbitrary frustration. Many authors (e.g., Buss, 1963) have stated that the experience of nonarbitrary frustration is weak and less likely to lead to aggression than frustration that is arbitrary or unjustified.

The self-report measures of mood were associated with some of the competitive behaviors. The fact that subjects rated themselves as both more tense and more angry if they had shocked lends additional support to the validity of the shock response as an indicant of aggression in the competition procedure (Hynan \& Esselman, Note 1). Also, the subject's level of self-reported frustration was higher if the subject was losing. This suggests that a manipulation of the probability of winning may be a more potent determinant of frustration.

\section{EXPERIMENT 2}

In Experiment 2, the subject's chances of winning were manipulated by varying the rate of accumulation of points for the research assistant. Subjects were assigned to one of three conditions (win, tie, and lose). It was expected that both level of frustration and shock usage would be inversely related to the subject's chances of winning.

\section{Method}

Subjects. Data were obtained from 30 male and 30 female University of Wisconsin-Milwaukee undergraduates.

Apparatus. All materials from Experiment 1 were again used. The POMS was scored on all scales (anger, tension, confusion, 
depression, vigor, and fatigue). Four females and one male served as research assistants.

Procedure. The basic procedure used was that of the $12+R$ condition of Experiment 1 . Subjects in the win condition competed against an opponent who accumulated points on a VT 5.1point $/ \mathrm{sec}$ schedule (range $=3.1-7.1$ points $/ \mathrm{sec}$ ). The schedules in the tie and lose conditions were VT 6.4 points $/ \mathrm{sec}$ (range $=4.5$ 8.3 points $/ \mathrm{sec}$ ) and VT 8.8 points $/ \mathrm{sec}$ (range $=6.1-11.5$ points $/ \mathrm{sec}$ ), respectively. Subjects were also allowed to shock with their left hands, in an attempt to raise the baseline of shocking. The righthand restriction for point accumulation and blocking was still in effect.

\section{Results}

All of the subjects in the win condition, $70 \%$ of the subjects in the tie condition, and none of the subjects in the lose condition won the game $\left[\chi^{2}(2)=\right.$ $112.8, \mathrm{p}<.01]$. The nominal criterion of shocking was not related to the manipulation of winning. Shock was delivered by eight males and seven females (nonsignificant).

Shock frequency and block frequency scores were analyzed by a 2 (sex) by 3 (win condition) by 3 (game period) multivariate analysis of variance. The multivariate test of the Win Condition by Game Period interaction approached significance $[\mathrm{F}(8,102)=1.76$, $\mathrm{p}<.10$ ]. Univariate follow-up tests revealed that an interaction occurred across the linear function of block scores $[\mathrm{F}(1,54)=4.98, \mathrm{p}<.025]$. Figure 2 shows that while there were no differences in block frequency among groups during the first period of

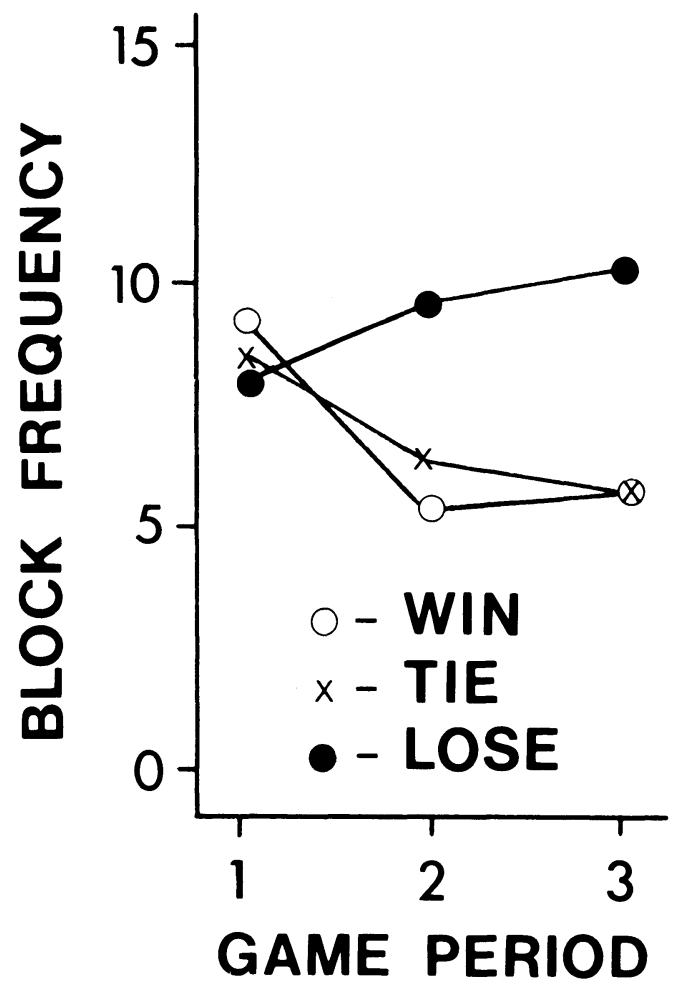

Figure 2. Mean block responses in the win, tie, and lose conditions across the three game periods. play, subjects in the lose condition blocked more than subjects in the tie and win conditions during the third period $[\mathrm{F}(1,54)=7.82, \mathrm{p}<.01]$.

A separate 2 (sex) by 3 (win condition) multivariate analysis of variance was applied to the seven mood scales. There was a significant main effect of the win conditions upon the mood states $[F(19,96)=$ $1.79, \mathrm{p}<.05$ ]. Follow-up tests showed that subjects who were in the lose condition rated themselves as being more frustrated (mean $=1.0)$ than did those in the win condition (mean $=.15)[F(1,54)=4.03$, $\mathrm{p}=.05]$, more confused (mean $=1.3$ ) than those in the win and tie conditions (mean $=.8)[\mathrm{F}(1,54)=$ $10.07, p<.01]$, and less vigorous (mean $=1.9$ ) than subjects in the tie condition (mean $=2.3)[\mathrm{F}(1,54)=$ $4.89, \mathrm{p}<.05]$. There were no differences in mood between those subjects who shocked and those who did not.

\section{Discussion}

The manipulation of winning was a more potent determinant of frustration than the manipulation of block frequency in Experiment 1 , yet in both cases aggressiveness was not affected. This was probably due to the low levels of frustration reported by subjects during the game. Even when subjects were blocked frequently and were losing, they stated that they were only a little frustrated. While this raises the question that perhaps subjects were apathetic about their participation, this view is not supported by other aspects of the data. Subjects in the losing condition had significant alterations in mood, and they blocked more frequently during the last period of the game, even as they fell further behind. Disinterested subjects would have slowed their rate of responding rather than speed up. Also, subjects were questioned concerning the amount of importance they felt concerning the outcome of the game. Subjects who won generally stated that it was important for them to win, whereas losers were more likely to state that it was just a game and not of great concern. It appears that the goal of winning the competitive game may not have been sufficiently compelling for the study of frustration effects. Any subsequent studies of the frustration-aggression hypothesis using this paradigm should increase the valence of winning.

Males and females shocked at equivalent rates during both experiments. This is consistent with past research using the competition paradigm (Hynan \& Esselman, Note 1), which has found that sex differences in aggressiveness are quite weak when levels of instigation are low (as in the two experiments presented here). Using higher levels of instigation in the competition paradigm, Hynan and Esselman found that males aggressed much more of ten than females. Thus, obtaining sex differences in aggression research may be related to the level of instigation used.

The failure of shock usage to covary with self-reported anger in Experiment 2 raises an issue concerning the validity of the shock response as an indicant of aggression. The validity of shocking in the competition paradigm may be dependent upon the procedures used in both Hynan and Esselman (Note 1) and in Experiment 1. In both instances, subjects had to abandon both blocking and scoring points in order to shock with the right hand. However, shocking was easier in Experiment 2, in that a procedural change allowed subjects to shock and block or to shock and score points simultaneously. This modification may have been responsible for the independence of anger and shocking in Experiment 2. This loss of validity indicates that care should be taken in aggression research that any procedural modification does not attenuate the validity of the shock response. Certainly, future research using the competition paradigm should maintain the physical incompatibility of the subject's responses. 


\section{REFERENCE NOTE}

1. Hynan, M. T., \& Esselman, J. Victims and aggression. Manuscript submitted for publication, 1980.

\section{REFERENCES}

Buss, A. Physical aggression in relation to different frustrations. Journal of Abnormal and Social Psychology, 1963, 67, 1-7.
KIRK, R. Experimental design: Procedures for the social sciences. Belmont, Calif: Brooks-Cole, 1968.

McNair, D., LorR, M., \& Droppleman, L. EDITS manual for the Profile of Mood States. San Diego: Educational and Industrial Testing Service, 1971.

(Received for publication September 7, 1980.) 